\title{
Game AI Competitions: Motivation for the Imitation Game-Playing Competition
}

\author{
Maciej Świechowski \\ QED Software \\ Warsaw, Poland \\ maciej.swiechowski@qed.pl \\ 0000-0002-8941-3199
}

\begin{abstract}
Games have played crucial role in advancing research in Artificial Intelligence and tracking its progress. In this article, a new proposal for game AI competition is presented. The goal is to create computer players which can learn and mimic the behavior of particular human players given access to their game records. We motivate usefulness of such an approach in various aspects, e.g., new ways of understanding what constitutes the human-like AI or how well it fits into the existing game production workflows. This competition may integrate many problems such as learning, representation, approximation and compression of AI, pattern recognition, knowledge extraction etc. This leads to multi-directional implications both on research and industry. In addition to the proposal, we include a short survey of the available game AI competitions.
\end{abstract}

\section{INTRODUCTION}

$\mathbf{E}$ VER since the inception of the first computers, making machines capable of playing games has been viewed as an opportunity to test their intelligence. Alan Turing was one of the pioneers of this idea [1]. The first games that became frameworks for Artificial Intelligence (AI) were checkers [2] and chess [3]. The latter has even been referred to as "The Drosophila of AI", because it has been studied extensively and this is a parallel to a type of fly in biology that was often featured in research. Games have become one of the most important testbeds for Aritifical Intelligence (AI). The main reasons are that they are relatively cheap, deterministic, easily repeatable and controllable as well as enterntaining testing environments. Moreover, many problems encountered in games, reflect some real-world problems, which is especially significant in modern video games.

We have gone a long way from those early research to famous competitions between a man and a machine. The most notable ones were IBM's Deep Blue triumph over Garry Kasparov [4] in chess and IBM Watson winning against human champions in Jeopardy! [5]. Research in computer chess after the last Ultimate Computer Chess Challenge [6] in 2007 shifted towards deep learning [7] and human-like approaches. More recently, one of the major breakthroughs, not only in games but in AI in general [8], was highlighted with the match between Lee Sedol and Google DeepMind's AlphaGo [9]

Research co-funded by the Smart Growth Operational Programme 20142020, financed by the European Regional Development Fund under GameINN project POIR.01.02.00-00-0184/17, operated by the National Centre for Research and Development (NCBR). in Go. For decades, computers could defeat the top human players only in simpler abstract games. Many of them have been solved, e.g. Connect-4 [10] and Checkers [11]. However, with successes of DeepMind's AlphaStar [12] in Starcraft and OpenAI Dota-Five [13] in Dota, computer bots have finally started to achieve human-level performance in video games too.

In this paper, we propose a new type of competition for game-playing bots. The main idea is to construct computer programs that are capable of playing a game in the most similar way to a given human player. There are numerous reasons of why we think it is useful for research community and game development industry as well. The idea is based on both research experience related to game AI [14], [15], [16] as well as commercial experience in working with AI engine for game studios [17].

The next section is devoted to a short survey of the major modern competitions regarding game-based AI. In Section III, we present the motivation for the new competition. Finally, the last section concludes the paper.

\section{Annual Game AI Competitions}

In this section, we refer to the major modern competitions for AI in games. We focus on the goal of each competition. Readers interested in particular competitions are advised to follow the references given. There have not been suitable research references to the last two of the presented competitions, so we present the URLs instead.

General Game Playing (GGP) [15] - proposed by Stanford Logic Group [18], is one of the oldest on this list, being first hosted in 2005. It has been organized during either AAAI or IJCAI conferences. In 2006 and 2007, there was a $\$ 10 \mathrm{~K}$ prize available for the winner. The idea is to create computer programs capable of playing any finite, deterministic, synchronous games, even previously unknown ones. Our program, named MINI-Player [19], has reached the quarter-final level twice. The games are given in the so-called Game Description Language (GDL). Abstract combinatorial games have been used including mostly simple board games. Participating agents are given relatively short amount of time (e.g. 1 minute) for preparation before each match and some shorter time for each move (e.g. 20 seconds). The 
strongest players are based on the Monte Carlo Tree Search algorithm [20].

General Video Game AI (GVG-AI) [21] - first hosted in 2014, now part of the IEEE Conference on Games (CoG). The idea stemmed from GGP and both competitions share many similarities. The motivation is to develop universal, online learning-based methods with as little of game-dependent heuristics as possible. However, instead of combinatorial games as in GGP, GVG-AI employs simple video games. Most of them resemble the old games played on the Atari computer. They are represented in Video Game Description Language (VGDL), which has been inspired by GDL. Participating bots have 1 second for initialization and 40-50ms per each move, so the action is more fast-paced compared to GGP. However, similar techniques seem to work the best such as MCTS or Rolling Horizon Evolutionary Algorithms. Currently, the competition runs in a few tracks such as Single Player Planning Track, 2/N-Player Planning Track and Level Generation Track.

Arimaa Challenge [22] - Arimaa is a game designed to be playable with a standard set of chess but much more difficult for computer agents. However, for human players the game is not considered more difficult to play than chess despite having a much higher branching factor of approx. 17000. For reference, the branching factor of chess is 35 . The competition has been oficially held since 2004. There was a prize available for the authors of the computer programs to beat human experts, called defenders, using a standard, off-the-shelf hardware. The prize ranged from $\$ 10 \mathrm{~K}$ to $\$ 17.5 \mathrm{~K}$ depending on the year. The prize was first claimed in 2015 by the program named Sharp. The competition has been discontinued since 2020

Starcraft AI [23] - Starcraft is a very succesful real-time strategy game developed by Blizzard Entertainment. It has been particularly interesting for research community [24]. It is a popular e-sport game as well, what makes it even more appealing. The original full game of Starcraft is used thanks to the API made available for programmers, called $B W A P I$, to develop their own agents. The competition started in 2010. It has been organized alongside AIIDE and IEEE CIG competitions. The goal is to create strong Starcraft bots that are capable of both strategic and tactical reasoning, resource gathering, base building, managing build orders and battle micro-management. The organizes provide an open-source implementation of the agent called UAlbertaBot, which participated in all competitions so far and won in 2013. It has become both the entry point for new developers and the baseline to compare against. Despite the fact that this competition uses a specific game, unlike GGP and GVGGP, the winning solutions display a variety of techniques including multi-agent systems, MCTS, real-time planning, hierarchical task networks, graph searching algorithms, path-finding, dynamic scripting, neural networks, Q-learning, decision trees, and lots of heuristics or hard-coded strategies.

microRTS AI [25] - proposed as an alternative to Starcraft Competition with the goal of being more abstract and more accessible. Starcraft is a complex commercial game with a relatively difficult API to work with in order to create bots. Lots of setup is required, so the learning curve is steep. MicroRTS involves common challenges found in RTS games such as strategic and tactical reasoning, resource management, recruiting units, expanding bases etc. Another difference to Starcraft is that the agents have access to a forward model (a simulator) of the game. The goal is to create a bot that is able to defeat the enemies. The winners display an interesting blend of techniques such as game-tree search, fast heuristics, grammars, dynamic scripting and, more recently, machine learning.

Fighting Game AI [26] - this competition uses an abstract representation of fighting games such as Mortal Kombat or Street Fighter. The game is played asynchronously in real time. The AI has maximum of $16.67 \mathrm{~ms}$ per frame to make a decision. There are 56 possible actions such as high punch, low kick, or block. The succesful agents are based on techniques such as Monte Carlo Tree Search, evolutionary algorithms or hierarchical reinforcement learning [27].

Visual Doom AI Competition (VizDoom) [28] - this AI platform is based on an old first-person shooter (FPS) game called Doom, which is now open-source. It is a very unique challenge as bots are given raw pixels (i.e., what the player sees) instead of some form of structured state representation as in the case of other game AI competitions. The agents have to reason about the surroundings, navigate through the levels, find interesting spots and weapons and fight with the opponents. There are two tracks of the competition. In the first one, the goal is to finish the game level in the shortest time. The second track is a typical deathmatch, in which the goal is to kill as many enemies as possible. This competition is advertised to be a benchmark for reinforcement learning agents [29]. The state-of-the-art techniques are based on deep learning [30]. However, even the most successful bots cannot compete with humans yet.

Hanabi [31] - Hanabi is a cooperative card game created by French game designer Antoine Bauza. The goal of the Hanabi AI Competition, hosted alongside IEEE CoG, is to create bots that can cooperate and win. The players have the option to give information, play a card or discard a card. Imperfect information plays crucial role in this game and therefore it is challenging for computer players [32]. It is considered a new AI framework for research in multi-agent learning. The agents submitted to the competition use various techniques such as Monte Carlo Tree Search, neural networks, reinforcement learning and rule-based systems.

Hearthstone AI [14] - Hearthstone is an extremely 
popular online collectible card game developed by Blizzard Entertainment. The first competition was run during IEEE CIG in 2018. It had the biggest number of entries among all competitions held during CIG. The agents played with predefined decks and were pitted against each other. Therefore, this is another example of a competition aimed at creating as strong computer players as possible in a particular game. Competitions in popular games such as Hearthstone have additional value to them, e.g. bots can generate data for learning-based algorithms aimed at solving particular game-related problems [33].

Strategy Card Game AI [34] - proposed as an alternative to Hearthstone just like microRTS competition has been introduced as a simpler alternative to Starcraft. This competition is based around a game called Legends of Code and Magic (LOCM). It is a small and relatively generic framework for research in the area of collectible card-games. The first installment of the competition was run in 2019. The bots are required to be capable of drafting cards (building a deck) as well as playing them.

Geometry Friends [35] - this is a two-player cooperative puzzle platformer game. One player plays as a circle, that can jump and roll and the other one as a rectangle that can change shape (but preserving the area) and slide. Physics with gravity and friction plays an important role. The goal is to complete levels and collect all diamonds that are placed on each level. The main challenges are motion planning and cooperation. The competition has been held in association with three different conferences in various years - IEEE CIG, Genetic and Evolutionary Computation (GECCO) and EPIA Conference on Artificial Intelligence.

Bot Bowl [36] - proposed in 2018 and based on the board game Blood Bowl. This sport game draws inspirations from fantasy and football. Agents have to control 11 players, which poses a challenge of having multiple actions to perform in a coordinated fashion. The competition uses Fantasy Football AI framwork written in Python. It was designed with the Python's machine learning ecosystem in mind. Currently, held during IEEE CoG.

Angry Birds Level Generation [37] - although this
competition is built upon a game framework, it has a distinct
goal - to create interesting and fun levels in the game of Angry
Birds. It belongs to the area of procedural content generation
(PCG). The winners of this competition predominantly rely
on methods such as evolutionary approaches [38].

Generative Design in Minecraft Competition [39] - like the previous competition, this one is not about the AI for bots, but rather procedural content generation. It is held as part of The Foundations of Digital Games (FDG) conference. The framework of choice is Minecraft - an extremely popular game based on voxels and using them to build objects, houses, settlements, complete maps. The goal of the competition is to use algorithms to produce content which will be both aesthetically pleasing and will evoke an interesting narrative. The evaluation is performed by human judges. That is an interesting fact which usually distinguishes PCG competitions from game-playing AI competitions. If there existed an acceptable way of automated evaluation of maps, then the PCG could directly employ it as part of the techniques, e.g. as fitness function in the evolutionary algorithm.

Halite Competition - available at https://halite.io/. This is both an AI competition and a programming contest. Halite is a resource management game. The goal is to create computer players that gather resources and navigate through $2 \mathrm{D}$ game map more effectively than their opponents. The authors of the competition report that participants from over 100 countries played more than 4 million games.

BattleCode - available at https://www.battlecode.org/. A competition hosted by MIT with $\$ 30 \mathrm{~K}$ tournament prize pool. Battlecode is a two-player real-time strategy game. It is both an AI challenge and programming competition aimed for student teams. This competition is not specially designed to advance the state-of-the-art in AI. The computer agents have to solve problems such as resource management, positioning, pathfinding, communication, finding proper offensive strategies.

\section{IMItATION GAME AI COMPETITION}

\section{A. Description}

We think that a new competition should be based around making computer bots that can effectively imitate any given human player. The term "imitation" requires elaborated definition. There are two viable approaches to measure the rate of imitation. The first one, more suitable for bigger competitions, is to do it in the same way as it is done in data-mining competitions, e.g. by having training data and hidden testing data. Examples of such data-mining competition platforms are Kaggle [40] and KnowledgePit [41]. The second one, has even featured game-based competition e.g. aimed at advising players [42] in card games. The second option, suitable for competitions with lesser amounts of entries, would be to have human referees judging the bots as shown in the Generative Design in Minecraft Competition. Although with this approach there is lesser objectivity, the human experts could see nuances that are difficult to grasp with automatic verification. It is also useful for the game industry - where all it matters is whether the bots' behavior feels right and whether the bots act the way game designers have envisioned.

The second aspect of the competition is how the input (training) data should be provided. The training data are past game records of the player to be imitated. They can have the form of videos, sequence of screenshots or structured logs. A particularly suitable format for such $\operatorname{logs}$ is in $\operatorname{LogDL}$ [43], which was inspired by GDL mentioned in the context of 
General Game Playing. We think that the most important thing is to not give the bots any more information than the human players had. Apart from that, they should be given as much information as possible amongst the information human players can see. We propose to combine the video footage with logs containing the most important numerical parameters that describe the game. This is a multi-modal approach. In this way, the bots can do feature extraction and figure out what describes the style of a particular player the most accurately. At the same time, they do not have to do the basic necessary extraction of the obvious parameters such as the amount of resources in strategy games. Therefore, video recognition methods could be focused on patterns, maps and geometric dependencies.

\section{B. Advantages}

In this section, we present the motivation behind the Imitation Game AI. After introducing each reason, we put annotations: Research or Industry in brackets. The former indicates that the particular reason mostly concerns advancing the state-of-the-art of the Artificial Intelligence field. The latter refers to advantages for the game development industry.

1) Human-likeness (Research, Industry): What makes human-like AI is a question that has been asked by many researchers, e.g. [44]. Although it is an interesting concept by itself from the cognitive viewpoint, it also has practical implications, e.g. in trusted human-robot interaction [45]. A competition that revolves around this topic would not only spawn new methods of implementing human-like AI, but also new ways of measuring it. Such methods could be more objectivized that those that are based on the Turing's Test as they would rely less on the judgement performed by a relatively small number of referees.

Human-like bots are very valuable for computer games as well for numerous reasons. First of all, they can act as believable NPC characters [46]. This greatly increases the immersion in the game. More immersive and interesting games lead to more amount of time spent by people playing them and a better overall reception. Second of all, they can take part in multi-player matches if there are not enough human players available at given moment or they can take over when one of the human players disconnects from the game. Third of all, human-like bots can be used as virtual testers specialized to predict interactions real (human) players will make. If a goal of the AI challenge is to develop methods and techniques that can accurately capture the style of play of humans, then the property of human-likeness is an inherent part of the challenge.

2) Explainability (Research, Industry): As applications of machine learning models such as deep neural networks are growing in numbers, the explainable $\mathrm{AI}$ (XAI) is becoming more and more important [47]. In the game industry, the most commonly implemented AI techniques such as Behavior Trees, Finite State Machines or Utility AI are still highly explainable and interpretable. However, they are relatively limited in terms of complexity and competence levels of the AI. It seems inevitable that methods such as deep reinforcement learning will be applied more often not only in research but in shipped video games too. When the goal of the AI is to mimic specific human players, then if a bot achieves high accuracy regardless of how much of a black-box it is, we can explain it by asking the same human players for explanation of their reasoning. Expert human players tend to make thoughtful actions aimed at gaining some particular advantages in the game.

3) Controlled Difficulty (Industry): Superhuman bots are unacceptable in commercial video games. After all, the games need to be entertaining and possible to complete. On the other end of the spectrum, it is often difficult to create competent bots without letting them "cheat", e.g. have access to hidden information or gather resources more effectively than human players. The idea of mimicking human skills is a solution to both superhuman or incompetent bots. Here an important distinction can be seen between the goal of imitation of human skill in the game and just using human players as teachers in supervised learning. The latter case may, by chance, lead to the superhuman level of play. However, in our proposal, not surpassing human skill level inherently goes along with the aim of reproducing their skill.

4) Personification of AI (Industry): A persona system for bots means that even if multiple computer players are meant to have similar intelligence, they have certain individual characteristics, e.g., risk taker, explorer, conqueror, defender, builder etc. Therefore, they display various behaviors and are, in general, more interesting characters in games. Algorithms that are aimed at optimizing a value function such as Monte Carlo Tree Search or neural networks tend to converge (with faster or slower rate) to their optimality conditions. In order to introduce more variety with techniques such as MCTS, it was proposed to define game logic with varying granularity [48] Here comes an important property of our Imitation Game AI Competition, i.e., the requirement to be able to learn and mimic the behavior of particularly chosen human players. This is a different case than learning based on a general corpus of game records played by humans as this way we would lose the individual traits.

5) Commercially Viable Workflow (Industry): Human game testers are usually the biggest group of people involved in the game production process [49]. In fact, when a game requires complex $\mathrm{AI}$, sometimes it would be easier and cheaper to train it by letting it observe human players. It takes a lot of time of experienced AI designers and programmers to create a competent $\mathrm{AI}$ in games, where it plays an important role, e.g. shooter games with bots or strategy games. The Imitation Game AI competition solves enables an alternative way of coming up with the problem of creating the AI. Moreover, this approach fits into the existing game production pipeline, because games are thoroughly tested by people playing it, so many records of the played 


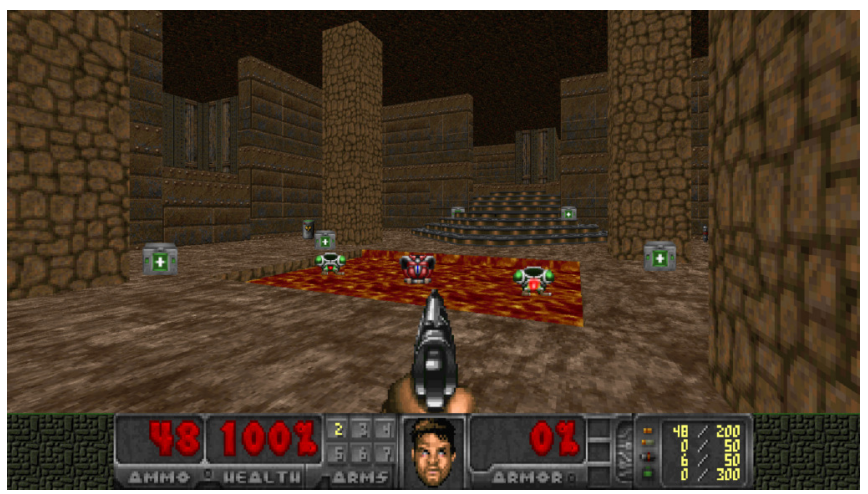

Fig. 1. Raw pixels shown to bots in the VizDoom Competition.

games are produced anyway for the internal companies' usage.

6) Vision Recognition (Research): The Imitation Game AI competition can be run with various forms of game records for the bots to observe and learn. One possibility is to give the bots raw pixels of the screen, just like humans observed the game. This is a similar case to the Visual Doom AI competition mentioned in Section II as shown in Figure 1. The other possibility is to provide structured logs of game records or even multi-modal data, e.g., combination of pixels, high-level representation of game states and data about the UI input state (mouse, keyboard, pad). In the first case, i.e., with raw visual data, the competition would benefit to the computer vision field. It would require effective methods of image classification [50], concepts extraction [51], object recognition [52] and visual reasoning [53].

7) Step Towards AGI (Research): Developing Artificial General Intelligence (AGI or "Strong AI") [54] is one of the distant challenges for the AI researchers. First and foremost, general AI competitions such GGP and GVG-AI with goals of creating universal game playing programs fit well into the AGI stream of research. We argue that the Imitation Game AI competition, in its general variant, is a step closer to AGI than a competition that revolves around making bots as strong as possible. AGI is not about making AI do something more effectively than humans - that is Artificial Superintelligence (ASI). The aim of AGI is to make an AI that can learn, reason and perform any task on par with humans. The focus is on generality in contrast to specialized Artificial Narrow Intelligence (ANI or "Weak AI").

\section{CONCLUSiONS}

In this paper, we proposed an AI benchmark competition that is aimed at creating bots capable of learning from humans to mimic their skill and style of play. Such an approach to learning is similar to supervised-learning. However, in a typical supervised-learning scenario, training data must be properly prepared and labelled. We leave it as an open problem to solve by the bot, i.e., the algorithms must figure out how to extract useful knowledge from the observed human players. Moreover, the the players that produce the training data and the bots have various objectives. Human players play according to the goals of the particular game. The goal for the bots is to play in a similar way to the human player. This can be particular beneficial to video-game industry. Most games are tested for hundreds or even thousands of man-hours, so why not to take advantage of it and use the game records for training bots. Moreover, the problem of creating AI is transferred from AI specialists to specialized algorithms. Bots created this way can be, by definition, more human-like, can have different personas based on who trained them and can be more explainable for humans. Lastly, let's not forget that imitation learning brings interesting challenges and it is an interesting concept by itself. We are pursuing the goal of Artificial General Intelligence, but smaller steps have to be made first. Game-based AI challenges may continue being very useful for measuring progress in the field.

\section{REFERENCES}

[1] A. M. Turing, "Can a Machine Think," The World of Mathematics, vol. 4, pp. 2099-2123, 1956.

[2] A. L. Samuel, "Some Studies in Machine Learning Using the Game of Checkers," IBM Journal of Research and Development, vol. 3, no. 3 pp. 210-229, 1959, DOI=10.1147/rd.33.0210.

[3] A. Newell, J. C. Shaw, and H. A. Simon, "Chess-Playing Programs and the Problem of Complexity," IBM Journal of Research and Development, vol. 2, no. 4, pp. 320-335, 1958, DOI=10.1147/rd.24.0320.

[4] M. Newborn, Kasparov versus Deep Blue: Computer Chess Comes of Age. Springer Science \& Business Media, 2012, DOI=10.1007/978-1 4612-2260-6.

[5] D. Ferrucci, A. Levas, S. Bagchi, D. Gondek, and E. T. Mueller, "Watson: Beyond Jeopardy!" Artificial Intelligence, vol. 199, pp. 93105, 2013, DOI=10.1109/ICCI-CC.2013.6622216.

[6] M. Newborn, "2007: Deep Junior Deep Sixes Deep Fritz in Elista, 4-2," in Beyond Deep Blue. Springer, 2011, pp. 149-157.

[7] D. Silver, T. Hubert, J. Schrittwieser, I. Antonoglou, M. Lai, A. Guez M. Lanctot, L. Sifre, D. Kumaran, T. Graepel et al., "A General Reinforcement Learning Algorithm that Masters Chess, Shogi, and Go Through Self-Play," Science, vol. 362, no. 6419, pp. 1140-1144, 2018, DOI=10.1126/science.aar6404.

[8] F.-Y. Wang, J. J. Zhang, X. Zheng, X. Wang, Y. Yuan, X. Dai, J. Zhang, and L. Yang, "Where does AlphaGo Go: from Church-Turing Thesis to AlphaGo Thesis and Beyond," IEEE/CAA Journal of Automatica Sinica vol. 3, no. 2, pp. 113-120, 2016, DOI=10.1109/JAS.2016.7471613.

[9] D. Silver, J. Schrittwieser, K. Simonyan, I. Antonoglou, A. Huang, A. Guez, T. Hubert, L. Baker, M. Lai, A. Bolton et al., "Mastering the Game of Go Without Human Knowledge," Nature, vol. 550, no. 7676, pp. 354-359, 2017, DOI=10.1038/nature24270.

[10] H. J. Van Den Herik, J. W. Uiterwijk, and J. Van Rijswijck, "Games Solved: Now and in the Future," Artificial Intelligence, vol. 134, no. 1-2, pp. 277-311, 2002, DOI=10.1016/S0004-3702(01)00152-7.

[11] J. Schaeffer, N. Burch, Y. Björnsson, A. Kishimoto, M. Müller, R. Lake, P. Lu, and S. Sutphen, "Checkers is solved," Science, vol. 317, no. 5844, pp. 1518-1522, 2007, DOI=10.1126/science.1144079.

[12] O. Vinyals, I. Babuschkin, W. M. Czarnecki, M. Mathieu, A. Dudzik, J. Chung, D. H. Choi, R. Powell, T. Ewalds, P. Georgiev et al., "Grandmaster Level in StarCraft II using Multi-Agent Reinforcement Learning," Nature, vol. 575, no. 7782, pp. 350-354, 2019, DOI=10.1038/s41586019-1724-z.

[13] S. McCandlish, J. Kaplan, D. Amodei, and O. D. Team, "An Empirical Model of Large-Batch Training," arXiv preprint arXiv:1812.06162, 2018.

[14] M. Świechowski, T. Tajmajer, and A. Janusz, "Improving Hearthstone AI by Combining MCTS and Supervised Learning Algorithms," in 2018 IEEE Conference on Computational Intelligence and Games (CIG). IEEE, 2018, pp. 1-8, DOI=10.1109/CIG.2018.8490368. 
[15] M. Świechowski, H. Park, J. Mańdziuk, and K.-J. Kim, "Recent Advances in General Game Playing," The Scientific World Journal, vol. 2015,2015 , DOI=10.1155/2015/986262.

[16] M. Świechowski and J. Mańdziuk, "Specialized vs. Multi-Game Approaches to AI in Games,' in Intelligent Systems' 2014. Springer, 2015, pp. 243-254, DOI=10.1007/978-3-319-11313-5 23.

[17] M. Świechowski and D. Ślęzak, "Grail: A Framework for Adaptive and Believable AI in Video Games," in 2018 IEEE/WIC/ACM International Conference on Web Intelligence (WI). IEEE, 2018, pp. 762-765, DOI=10.1109/WI.2018.00012.

[18] M. R. Genesereth, N. Love, and B. Pell, "General Game Playing: Overview of the AAAI Competition," AI Magazine, vol. 26, no. 2, pp. 62-72, 2005, DOI=10.1609/aimag.v26i2.1813.

[19] M. Świechowski and J. Mańdziuk, "Self-Adaptation of Playing Strategies in General Game Playing," IEEE Transactions on Computational Intelligence and AI in Games, vol. 6, no. 4, pp. 367-381, Dec 2014, DOI=10.1109/TCIAIG.2013.2275163.

[20] C. B. Browne, E. Powley, D. Whitehouse, S. M. Lucas, P. I. Cowling, P. Rohlfshagen, S. Tavener, D. Perez, S. Samothrakis, and S. Colton, "A Survey of Monte Carlo Tree Search Methods," IEEE Transactions on Computational Intelligence and AI in Games, vol. 4, no. 1, pp. 1-43, 2012, DOI=10.1109/TCIAIG.2012.2186810.

[21] D. Perez-Liebana, S. Samothrakis, J. Togelius, T. Schaul, S. M. Lucas, A. Couëtoux, J. Lee, C.-U. Lim, and T. Thompson, "The 2014 General Video Game Playing Competition," IEEE Transactions on Computational Intelligence and AI in Games, vol. 8, no. 3, pp. 229-243, 2015 DOI=10.1109/TCIAIG.2015.2402393.

[22] O. Syed and A. Syed, Arimaa - A New Game Designed to be Difficult for Computers. Institute for Knowledge and Agent Technology, 2003, vol. 26, no. 2, DOI=10.3233/icg-2003-26213.

[23] S. Xu, H. Kuang, Z. Zhi, R. Hu, Y. Liu, and H. Sun, "Macro Action Selection with Deep Reinforcement Learning in Starcraft," in Proceedings of the AAAI Conference on Artificial Intelligence and Interactive Digital Entertainment, vol. 15, no. 1, 2019, pp. 94-99.

[24] S. Ontanon, G. Synnaeve, A. Uriarte, F. Richoux, D. Churchill, and M. Preuss, "A Survey of Real-Time Strategy Game AI Research and Competition in StarCraft," IEEE Transactions on Computational Intelligence and AI in Games, vol. 5, no. 4, pp. 293-311, 2013, DOI=10.1109/TCIAIG.2013.2286295.

[25] S. Ontañón, N. A. Barriga, C. R. Silva, R. O. Moraes, and L. H. Lelis, "The First MicroRTS Artificial Intelligence Competition," AI Magazine, vol. 39, no. 1, pp. 75-83, 2018, DOI=10.1609/aimag.v39i1.2777.

[26] Y. Takano, H. Inoue, R. Thawonmas, and T. Harada, "SelfPlay for Training General Fighting Game AI," in 2019 Nicograph International (NicoInt). IEEE, 2019, pp. 120-120, DOI=10.1109/NICOInt.2019.00034

[27] I. P. Pinto and L. R. Coutinho, "Hierarchical Reinforcement Learning with Monte Carlo Tree Search in Computer Fighting Game," IEEE Transactions on Games, vol. 11, no. 3, pp. 290-295, 2018 , DOI=10.1109/TG.2018.2846028.

[28] M. Wydmuch, M. Kempka, and W. Jaśkowski, "Vizdoom Competitions: Playing Doom from Pixels," IEEE Transactions on Games, vol. 11, no. 3 , pp. 248-259, 2018, DOI=10.1109/TG.2018.2877047.

[29] M. Kempka, M. Wydmuch, G. Runc, J. Toczek, and W. Jaśkowski, "Viz doom: A Doom-based AI Research Platform for Visual Reinforcement Learning," in 2016 IEEE Conference on Computational Intelligence and Games (CIG). IEEE, 2016, pp. 1-8, DOI=10.1109/CIG.2016.7860433.

[30] K. Shao, D. Zhao, N. Li, and Y. Zhu, "Learning Battles in ViZDoom via Deep Reinforcement Learning," in 2018 IEEE Conference on Computational Intelligence and Games (CIG). IEEE, 2018, pp. 1-4, DOI=10.1109/CIG.2018.8490423.

[31] N. Bard, J. N. Foerster, S. Chandar, N. Burch, M. Lanctot, H. F. Song, E. Parisotto, V. Dumoulin, S. Moitra, E. Hughes et al., "The Hanab Challenge: A New Frontier for AI Research," Artificial Intelligence, vol. 280, p. 103216, 2020, DOI=10.1016/j.artint.2019.103216.

[32] J.-F. Baffier, M.-K. Chiu, Y. Diez, M. Korman, V. Mitsou, A. van Renssen, M. Roeloffzen, and Y. Uno, "Hanabi is NP-hard, even for cheaters who look at their cards," Theoretical Computer Science, vol. 675, pp. 43-55, 2017, DOI=10.1016/j.tcs.2017.02.024.

[33] A. Janusz, Ł. Grad, and D. Ślęzak, "Utilizing Hybrid Information Sources to Learn Representations of Cards in Collectible Card Video Games," in 2018 IEEE International Conference on Data Mining Workshops, ICDM Workshops, Singapore, Singapore, November 17-20, 2018. IEEE, 2018, pp. 422-429, DOI=10.1109/ICDMW.2018.00069.
[34] J. Kowalski and R. Miernik, "Evolutionary Approach to Collectible Card Game Arena Deckbuilding using Active Genes," Accepted to IEEE Congress on Evolutionary Computation 2020, 2020. [Online]. Available: arXivpreprintarXiv:2001.01326

[35] D. M. G. Verghese, S. Bandi, and G. J. Jayaraj, "Solving the Complexity of Geometry Friends by Using Artificial Intelligence," in Advances in Decision Sciences, Image Processing, Security and Computer Vision. Springer, 2020, pp. 528-533, DOI=10.1007/978-3-030-24318-0_62.

[36] N. Justesen, L. M. Uth, C. Jakobsen, P. D. Moore, J. Togelius, and S. Risi, "Blood Bowl: A New Board Game Challenge and Competition for AI," in 2019 IEEE Conference on Games (CoG). IEEE, 2019, pp $1-8$, DOI=10.1109/CIG.2019.8848063.

[37] J. Renz, X. Ge, S. Gould, and P. Zhang, "The Angry Birds AI competition," AI Magazine, vol. 36, no. 2, pp. 85-87, 2015, DOI=10.1609/aimag.v36i2.2588.

[38] A. Irfan, A. Zafar, and S. Hassan, "Evolving Levels for General Games Using Deep Convolutional Generative Adversarial Networks," in 2019 11th Computer Science and Electronic Engineering (CEEC). IEEE, 2019, pp. 96-101, DOI=10.1109/CEEC47804.2019.8974332.

[39] C. Salge, M. C. Green, R. Canaan, and J. Togelius, "Generative Design in Minecraft (GDMC) Settlement Generation Competition," in Proceedings of the 13th International Conference on the Foundations of Digital Games, 2018, pp. 1-10.

[40] J. Whitehill, "Climbing the Kaggle Leaderboard by Exploiting the LogLoss Oracle," in Workshops at the Thirty-Second AAAI Conference on Artificial Intelligence, 2018

[41] A. Janusz, D. Slezak, S. Stawicki, and M. Rosiak, "Knowledge Pit - A Data Challenge Platform," in CS\&P, 2015, pp. 191-195.

[42] A. Janusz, T. Tajmajer, M. Świechowski, Ł. Grad, J. Puczniewski, and D. Ślęzak, "Toward an Intelligent HS Deck Advisor: Lessons Learned from AAIA'18 Data Mining Competition," in 2018 Federated Conference on Computer Science and Information Systems (FedCSIS). IEEE, 2018, pp. 189-192, DOI=10.15439/2018F386.

[43] M. Swiechowski and D. Slęzak, "Introducing LogDL - Log Description Language for Insights from Complex Data," in Proceedings of the 15th Federated Conference on Computer Science and Information Systems (FedCSIS). IEEE, 2020, pp. 145-154.

[44] S. D. Baum, B. Goertzel, and T. G. Goertzel, "How Long until Human-Level AI? Results from an Expert Assessment," Technological Forecasting and Social Change, vol. 78, no. 1, pp. 185-195, 2011, DOI=10.1016/j.techfore.2010.09.006.

[45] J. Fink, "Anthropomorphism and Human Likeness in the Design of Robots and Human-Robot Interaction," in International Conference on Social Robotics. $\quad$ Springer, 2012, pp. 199-208, DOI=10.1007/978-3642-34103-8_20.

[46] P. Hingston, Believable Bots: Can Computers Play Like People? Springer, 2012, DOI=10.1007/978-3-642-32323-2

[47] J. Zhu, A. Liapis, S. Risi, R. Bidarra, and G. M. Youngblood, "Explainable AI for designers: A human-centered perspective on mixed-initiative co-creation," in 2018 IEEE Conference on Computational Intelligence and Games (CIG). IEEE, 2018, pp. 1-8, DOI $=10.1109 /$ CIG.2018.8490433.

[48] M. Świechowski and D. Ślęzak, "Granular Games in RealTime Environment," in 2018 IEEE International Conference on Data Mining Workshops (ICDMW). IEEE, 2018, pp. 462-469, DOI=10.1109/ICDMW.2018.00074

[49] D. Irish, The game poducer's handbook. Course Technology Press, 2005, DOI=10.5555/1209055.

[50] J. Wang, Y. Yang, J. Mao, Z. Huang, C. Huang, and W. Xu, “CNNRNN: A Unified Framework for Multi-label Image Classification,” in Proceedings of the IEEE Conference on Computer Vision and Pattern Recognition, 2016, pp. 2285-2294, DOI=10.1109/CVPR.2016.251.

[51] N. A. Bennett, Q. He, C. Chang, and B. R. Schatz, "Concept Extraction in the Interspace Prototype," University of Illinois at UrbanaChampaign, Champaign, IL, 1999, DOI $=10.5555 / 871248$.

[52] R. M. Cichy, D. Pantazis, and A. Oliva, "Resolving Human Object Recognition in Space and Time," Nature Neuroscience, vol. 17, no. 3 , p. 455,2014 , DOI $=10.1038 / \mathrm{nn} .3635$.

[53] C. Han, J. Mao, C. Gan, J. Tenenbaum, and J. Wu, "Visual ConceptMetaconcept Learning," in Advances in Neural Information Processing Systems, 2019, pp. 5002-5013.

[54] B. Goertzel, "Artificial General Intelligence: Concept, State of the Art, and Future Prospects," Journal of Artificial General Intelligence, vol. 5 , no. 1, pp. 1-48, 2014, DOI=10.2478/jagi-2014-0001. 